\title{
Correction to: Language, Script, and Font Recognition
}

\author{
Umapada Pal and Niladri Sekhar Dash
}

\section{Correction to: \\ Chapter 9 in: D. Doermann, K. Tombre (eds.), Handbook of Document Image Processing and Recognition, https://doi.org/10.1007/978-0-85729-859-1_9}

Owing to an unfortunate oversight the second author Niladri Sekhar Dash was missing in the initially published html version of this chapter. He has now been added. 